\title{
Characterization and functional analysis of pollen-specific PwSWEET1 in Picea wilsonii
}

\author{
Yanni Zhou $^{1} \cdot$ Xiaoyue Cui $^{1} \cdot$ Anni Hu${ }^{1} \cdot$ Yahui Miao $^{1} \cdot$ Lingyun Zhang $^{1}$
}

Received: 2 December 2017/Accepted: 4 November 2018/Published online: 11 June 2019

(C) The Author(s) 2019

\begin{abstract}
SWEET transporters play a pivotal role in sugar transport in plants. However, their functions in pollen tube growth, especially in coniferous species remain unknown. Here, we used RT-qPCR to reveal that a SWEETI gene was specifically expressed in pollen and pollen tubes of Picea wilsonii. A pollen germination assay showed that PwSWEETI was induced by $\mathrm{H}_{3} \mathrm{BO}_{3}$ but not by $\mathrm{Ca}^{2+}$. In a sugar specificity experiment, sucrose (Suc) and glucose (Glc) were effective sugars for pollen germination and pollen tube growth. PWSWEETI expression was induced most by Suc and Glc. Heterologous expression of PwSWEET1 in yeast showed that PwSWEET1 can restore the glucose absorption in yeast strain EBY.VW4000, which has a hexose absorption defect, and the absorption of glucose is pH-independent. This evidence supports the involvment of PwSWEET1 in boron-dependent glucose transport in pollen germination and pollen tube growth of Picea wilsonii.
\end{abstract}

Project funding: This work was supported by the grant from Agricultural Ministry of China (No. 2016ZX08009-003-002).

The online version is available at http://www.springerlink.com

Corresponding editor: Zhu Hong.

Electronic supplementary material The online version of this article (https://doi.org/10.1007/s11676-019-00986-x) contains supplementary material, which is available to authorized users.

Lingyun Zhang

lyzhang@bjfu.edu.cn; jody0826zhou@sina.com

1 Key Laboratory of Forest Silviculture and Conservation of the Ministry of Education, Beijing Forestry University, Beijing 100083, People's Republic of China
Keywords PwSWEET1 · Picea wilsonii · Pollen tube · Glucose transporter

\section{Introduction}

Sugars play essential roles as a main source of energy for many developmental processes in plants. Sugars are present mainly in the form of sucrose, glucose and fructose (Ap Rees 1994; Koch 1996). Sinks such as pollen grains, pollen tubes and the anther tapetum are usually completely isolated symplastically with no access to plasmodesmata (Buttner and Sauer 2000; McCormick 2004). The early developmental stage of pollen microspores and the subsequent pollen germination and pollen tube growth, however, absorb an enormous amount of nutrients from tapetal cells and female tissues, respectively (Ylstra et al. 1998; Ma 2005). Disruptions in carbohydrate supply or transport can irreversibly impair pollen development and pollen tube growth (Engelke et al. 2010; Ji et al. 2010). The large amounts of sugars and components required for these sinks must therefore be exported via the apoplast (Slewinski 2011), and photoassimilates can only be imported via specific transporters in the plasma membrane (Buttner and Sauer 2000). Chen et al. (2012) found that AtSWEET10-15 and OsSWEET11,-14 can transport sucrose across the plasma membrane to assist phloem loading in leaves and that they have lower transport activity for glucose. SWEETs may also function in carbohydrate transport during senescence; overexpression of AtSWEET15 can accelerate senescence, which may be related to carbohydrate supply in different organs (Seo et al. 2011).

SWEETs have been widely investigated in plants such as Arabidopsis, rice, and wheat (Yang et al. 2006; Guan et al. 2008; Song et al. 2009; Chen et al. 2010, 2012; 
Chardon et al. 2013; Guo et al. 2014; Xu et al. 2016) and have been divided into four subclades (Chen et al. 2010). Typical SWEETs proteins contain either seven transmembrane helices (TMs) or fewer than seven (Chen et al. 2010; Feng et al. 2015; Patil et al. 2015). They have also been reported to be involved in stress responses. In wild rice, infection with PXO99 ${ }^{\mathrm{A}}$ (Xanthomonas oryzae pv. oryzae) can induce the expression of OsSWEET11 to provide the bacteria with glucose (Chen 2014). In Arabidopsis, the pathogen Botrytis cinerea induces AtSWEET4, -15 and -17, and infection by Golovinomyces cichoracearum leads to increased expression of AtSWEET12 (Ferrari et al. 2007). SWEET responses to abiotic stress reported are related primarily to cold stress (Chardon et al. 2013; Klemens et al. 2013; Liu et al. 2016), and the cold stress response is always closely correlated with sugar accumulation (Ramirez et al. 2004; Ypsilos and Magan 2004; Ogawa et al. 2005).

Various researchers have raised awareness that sugar transporters are also involved in pollen development and pollen tube growth (Marger and Saier 1993; Ylstra et al. 1998; Johnson et al. 2006; Cheng et al. 2015). In cucumber, antisense suppression of $\mathrm{CsHTl}$ inhibits pollen germination and tube growth (Cheng et al. 2015). Arabidopsis thaliana mutants with a loss of AtSUC1 function are impaired in pollen germination (Sivitz et al. 2008). Inhibition of LeSUT2 in tomato leads to poorer germination and pollen tube growth, thus affecting fruit and seed development (Hackel et al. 2006). Also, several SWEETs related to pollen development have been reported. For example, pollen grains of atsweet 8 mutants of A. thaliana are aborted early in development (Guan et al. 2008). OsSWEET11-silenced rice plants have low pollen viability (Yang et al. 2006).

Due to species differences and the complexities of the physiological processes, the role of sugar transporters in pollen tube growth has remained poorly understood (Cheng et al. 2015). Compared with angiosperm pollen tubes, coniferous pollen tubes grow more slowly and tend to ramify with a lack of a tip-to-base organelle zonation ( $\mathrm{Yu}$ et al. 2009). Therefore, angiosperm pollen tubes are considered to be another evolutionary divergence of the male gametophytes (Lazzaro 2005); however, little information regarding this development is available. Here, we used RTqPCR to show that the gene SWEET1 from Picea wilsonii Mast. is specifically expressed in pollen and pollen tubes. Suc and Glc are the effective sugars for pollen germination of $P$. wilsonii and induce PwSWEET1 expression. Heterologous expression in a yeast strain defective in hexose absorption showed that PWSWEETI can restore the ability to absorb hexose.

\section{Materials and methods}

\section{Plant materials}

Pollen grains, cones and seeds were collected in 2014 from mature trees of $P$. wilsonii in the Beijing Botanical Garden, Chinese Academy of Sciences. Needles, stems and youngneedles were collected from trees on the campus of Beijing Forestry University. Pollen grains were dried overnight at room temperature, then stored at $-80{ }^{\circ} \mathrm{C}$ until use. Seedlings were cultivated for 8 weeks in a greenhouse at $25{ }^{\circ} \mathrm{C}$ with $16 \mathrm{~h}$ light/ $8 \mathrm{~h}$ dark.

\section{In vitro pollen germination}

Pollen grains stored at $-80{ }^{\circ} \mathrm{C}$ were revived by transferring to $4{ }^{\circ} \mathrm{C}$ for $24 \mathrm{~h}$, then at room temperature for $2 \mathrm{~h}$. The revived pollen grains were then cultured in different media. The standard liquid medium for pollen germination and tube growth was composed of $12 \%(\mathrm{w} / \mathrm{v})$ sucrose, $0.03 \%$ (w/v) $\mathrm{Ca}\left(\mathrm{NO}_{3}\right)_{2}, 0.01 \%(\mathrm{w} / \mathrm{v}) \mathrm{H}_{3} \mathrm{BO}_{3}$, and $5 \mathrm{mM}$ citratephosphate buffer ( $\mathrm{pH} 5.8)$. The effect of $\mathrm{B}$ and $\mathrm{Ca}$ on expression of PwSWEET1 was assessed using $0.1 \% \mathrm{Ca}^{2+}$ or $0.1 \% \mathrm{H}_{3} \mathrm{BO}_{3}$ in place of the standard concentrations in the standard liquid medium, then quantifying expression after $12 \mathrm{~h}$ and $24 \mathrm{~h}$. In the sugar specificity experiment, $3 \%, 8 \%, 12 \%$ and $18 \%$ (w/v) of a sugar (Suc, Glc, Gal, Mal, Fru, or Man) was added to the medium, then pollen tubes were examined after $24 \mathrm{~h}$. Pollen grains were incubated in small dishes at $25^{\circ} \mathrm{C}, 120 \mathrm{rpm} / \mathrm{min}$ in the dark and sampled at $6,12,18,24,30$ and $36 \mathrm{~h}$.

\section{Cloning of PwSWEET1 cDNA}

The shotgun method was used to obtain the full length sequence of PwSWEET1 cDNA based on the P. Wilsonii cDNA library constructed in our previous study (Zhang et al. 2012). Universal primers of pDONR222 vector $5^{\prime}$ GGT AAC GCC AGG GTT TTC C-3' (M13F) and 5'-CAG GAA ACA GCT ATG ACC-3' (M13R) were used.

\section{Bioinformatics analyses}

Nucleotide and amino acid sequences were analyzed using DNAMAN software. The homologous amino acid sequences of PwSWEET1 in Arabidopsis were searched from The Arabidopsis Information Resource (http://www. arabidopsis.org/). Amino acid sequences were aligned using Clustal $\mathrm{X}$, and the phylogenetic tree was constructed using MEGA5.0 software. The TMHMM v. 2.0 server (http://www.cbs.dtu.dk/services/TMHMM/) was used to predict transmembrane domains. 
Stress and hormone treatments of $\boldsymbol{P}$. wilsonii seedlings

The seedlings described in the Plant Materials section were used in this experiment. For salt stress, the roots of seedlings were treated with $200 \mathrm{mM} \mathrm{NaCl}$ for 1, 3, 6 and $12 \mathrm{~h}$. For cold stress and heat stress, the seedlings were respectively treated in refrigerator at $4{ }^{\circ} \mathrm{C}$ for 1,6 and $12 \mathrm{~h}$ and in water bath at $42{ }^{\circ} \mathrm{C}$ for $1,3,6$ and $12 \mathrm{~h}$. For drought stress, the seedlings were exposed to air without watering for 1,3 , $6,12 \mathrm{~h}$. For osmotic stress, the roots of seedlings were treated with $20 \mathrm{mM} \mathrm{H}_{2} \mathrm{O}_{2}$ for $1,3,6$ and $12 \mathrm{~h}$. For ABA treatment and MeJA treatment, the roots of seedlings were respectively treated with $100 \mu \mathrm{M}$ ABA for 3,6 and $12 \mathrm{~h}$ and $100 \mu \mathrm{M}$ MeJA for 1, 3, 6 and $12 \mathrm{~h}$. The control group $(0 \mathrm{~h})$ was cultivated in a greenhouse at $25{ }^{\circ} \mathrm{C}$ with $16 \mathrm{~h}$ light $/ 8 \mathrm{~h}$ dark. The treated seedlings were then frozen in liquid nitrogen and stored at $-80{ }^{\circ} \mathrm{C}$ until RNA was extracted. All of experiments were done three times.

\section{Quantitative RT-PCR}

PwSWEET1 expression was quantified using SYBR GREEN SuperReal Premix (Tiangen, Beijing) with a StepOnePlus Real-Time PCR System (ABI, USA) and specific primers for PwSWEETl (5'-GTGCGTTGTGGGTGTTAT$3^{\prime}$ and $5^{\prime}$-TCGTTTTTGTCCTTACAG-3'). EF1- $\alpha$ was amplified as an internal control using specific primers $5^{\prime}$ AACTGGAGAA GGAACCCAAG- $3^{\prime}$ and $5^{\prime}$-AACGACCCAATGGAG GATAC- $3^{\prime}$ (Yu et al. 2011). The RTqPCR was performed with $30-33$ cycles of $95{ }^{\circ} \mathrm{C}$ denaturation, $55{ }^{\circ} \mathrm{C}$ annealing and $72{ }^{\circ} \mathrm{C}$ extension and done independently three times.

\section{Functional characterization of PwSWEET1 by heterologous expression in yeast}

To test the biochemical properties of PwSWEET1, we constructed the plasmid combined with the CDS domain of PwSWEET1 in the yeast expression vector pDR196 (Fan et al. 2009). The primers was designed based on appropriate restriction sites (5'-SmaI, 3'-SalI) with forward primer: 5'-TCCCCCGGGA TGGCGAATACGGATACT-3' and reverse primer: $5^{\prime}$-TTCCGCGGCCGCTATGGCCGACGTCGACT CACGGAATTCCATTCCTATC- ${ }^{\prime}$. The PCR products were digested simultaneously with SmaI and SalI to combine with digested vector pDR196 to form the recombinant plasmid pDR196/PwSWEET1, and the construct was confirmed by PCR. The hexose transporter-deficient yeast strain EBY.VW4000 (Wieczorke et al. 1999) was transformed with pDR196/PwSWEET1, and a transformation with empty vector pDR196 was used as a control. The yeast uptake test was done as described by Chen et al. (2010) and Cheng et al. (2015). Cells were allowed to grow in liquid SD (lacking ura) supplemented with $2 \%$ (w/ v) maltose as the sole carbon source and harvested at $\mathrm{OD}_{600}$ 0.6. Different dilutions of the cell suspension were dropped on solid SD (lacking ura) (pH 5.0) supplemented with $2 \%(\mathrm{w} / \mathrm{v})$ maltose or Glc as the sole carbon source at different pHs. Cells were incubated $2-5 \mathrm{~d}$ at $29^{\circ} \mathrm{C}$ in the dark before photography.

\section{Results}

\section{Characterization of the cDNA clone for SWEET from $P$. wilsonii}

The 1237-nucleotide PwSWEETI cDNA encodes a 261-amino-acid polypeptide with an ATG initiation codon at position 100 and a termination codon at position 887 (TGA). To examine the evolutionary relationship among PwSWEET1, AtSWEETs and OsSWEETs, a phylogenetic analysis based on the amino acid sequences was conducted. PwSWEET1 was highly homologous with AtSWEET4, -5, -6 and -7 and OsSWEET4, -5, -6a, -6b, -7a, -7b, -7c, -7d and $-7 \mathrm{e}$, which belong to clade 2 in the SWEET superfamily (Fig. 1).

\section{Expression of PwSWEET1 in different tissues and in response to stresses and hormones}

As determined by RT-qPCR, PwSWEET1 was extensively expressed in the different organs and tissues, but pollen grains had the highest transcript level, up to 390-fold higher than in the needles and other tissues (Fig. 2a). Expression was also relatively high in needles, suggesting $P w S W E E T 1$ is probably involved in responses to stress and hormones (Fig. 2a). To further explore this involvement, we treated 8-week-old seedlings with salt $(\mathrm{NaCl})$, cold, heat, drought, $\mathrm{H}_{2} \mathrm{O}_{2}$, ABA and MeJA (Fig. 2). Under cold treatment $\left(4{ }^{\circ} \mathrm{C}\right), P w S W E E T 1$ expression increased and reached a maximum of 2.8 -fold at $1 \mathrm{~h}$, then declined at $6 \mathrm{~h}$ and increased again at $12 \mathrm{~h}$ (Fig. 2c). In contrast, the transcript level of PwSWEET1 decreased greatly at $1 \mathrm{~h}$ and declined to 0.08 -fold during heat treatment $\left(42{ }^{\circ} \mathrm{C}\right)$ (Fig. 2d). Drought treatment induced PwSWEET1 expression at $3 \mathrm{~h}$ to 2.1 -fold and $12 \mathrm{~h}$ to 2.5 -fold (Fig. 2e). Similarly, expression was also enhanced under $\mathrm{H}_{2} \mathrm{O}_{2}$ $(20 \mathrm{mM})$ treatment (Fig. 2f) and greatly increased in response to ABA $(100 \mu \mathrm{M})($ Fig. $2 \mathrm{~g})$ up to 15 -fold at $12 \mathrm{~h}$. Nevertheless, transcript levels did no obviously change during the salt $(200 \mathrm{mM})$ (Fig. 2b) and MeJA $(100 \mu \mathrm{M})$ treatments (Fig. 2h). Thus, we speculate that PwSWEETl very likely participates in $\mathrm{ABA}$ signal transduction and multiple stress responses. 
A

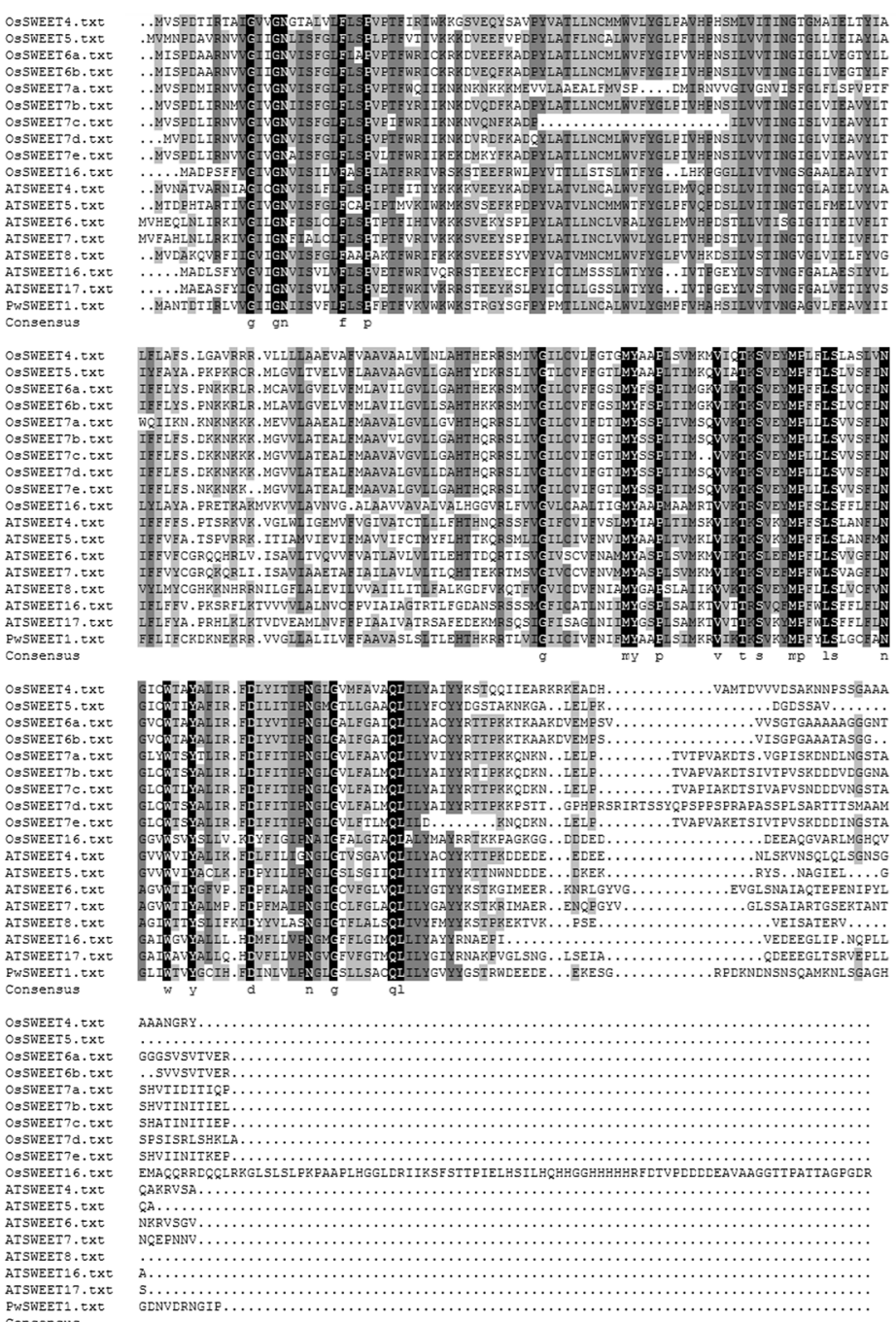

B

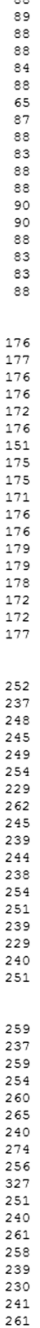

Fig. 1 The SWEETI gene of Picea wilsonii. a Amino acid sequence alignment of PwSWEET1 and SWEETs in Arabidopsis and rice. Different shades of gray represent different percentages of conservation (homology $=100 \%$ ), dark gray (homology $>75 \%$ ), light gray (homology $>50 \%$ ) and white (homology $>30 \%$ ); b phylogenetic tree analysis among PwSWEET1 and SWEETs in Arabidopsis and rice. This neighbor-joining tree was based on 1000 bootstrap replicates. Numbers on nodes are bootstrap values. Accession numbers: AtSWEET4
(AT3G28007), AtSWEET5 (AT5G62850), AtSWEET6 (AT1G66770), AtSWEET7 (AT4G10850), AtSWEET8 (AT5G40260), AtSWEET16 (AT3G16690), AtSWEET17 (AT4G15920), OsSWEET4 (Os02g19820), OsSWEET5 (Os05g51090), OsSWEET6a (Os01g42110), OsSWEET6b (Os01g42090), OsSWEET7a (Os09g08030), OsSWEET7b (Os09g08440), OsSWEET7c (Os12g07860), OsSWEET7d (Os09g08490), OsSWEET7e (Os09g08270), OsSWEET16 (Os03g22200)

early stage, suggesting PwSWEET1 is involved in pollen germination.

\section{$\mathrm{H}_{3} \mathrm{BO}_{3}$ induces PwSWEET1 expression}

Because $\mathrm{Ca}^{2+}$ and boron are involved in pollen germination and polarized pollen tube growth, we wondered whether there is a relationship among $\mathrm{Ca}^{2+}$, boron and $P$ WWEET1 expression. Considering that the optimal concentration of $\mathrm{Ca}^{2+}$ and boron for promoting pollen germination and polarized tube growth is, respectively, $0.03 \%(\mathrm{w} / \mathrm{v})$ and $0.01 \%(\mathrm{w} / \mathrm{v})(\mathrm{Yu}$ et al. 2011), we tested 
Fig. 2 Expression profiles of $P w S W E E T 1$ in different tissues and responses to stresses and hormones in 8-week-old Picea wilsonii seedlings. a Different tissues, b salt stress, c cold stress, $\mathbf{d}$ heat stress, e drought

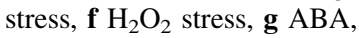
h MeJA

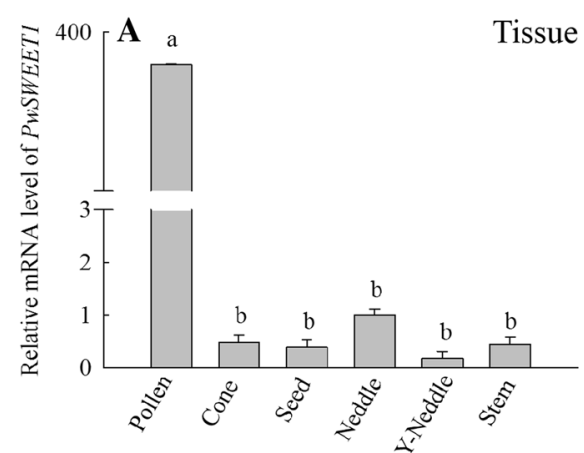

Different tissues
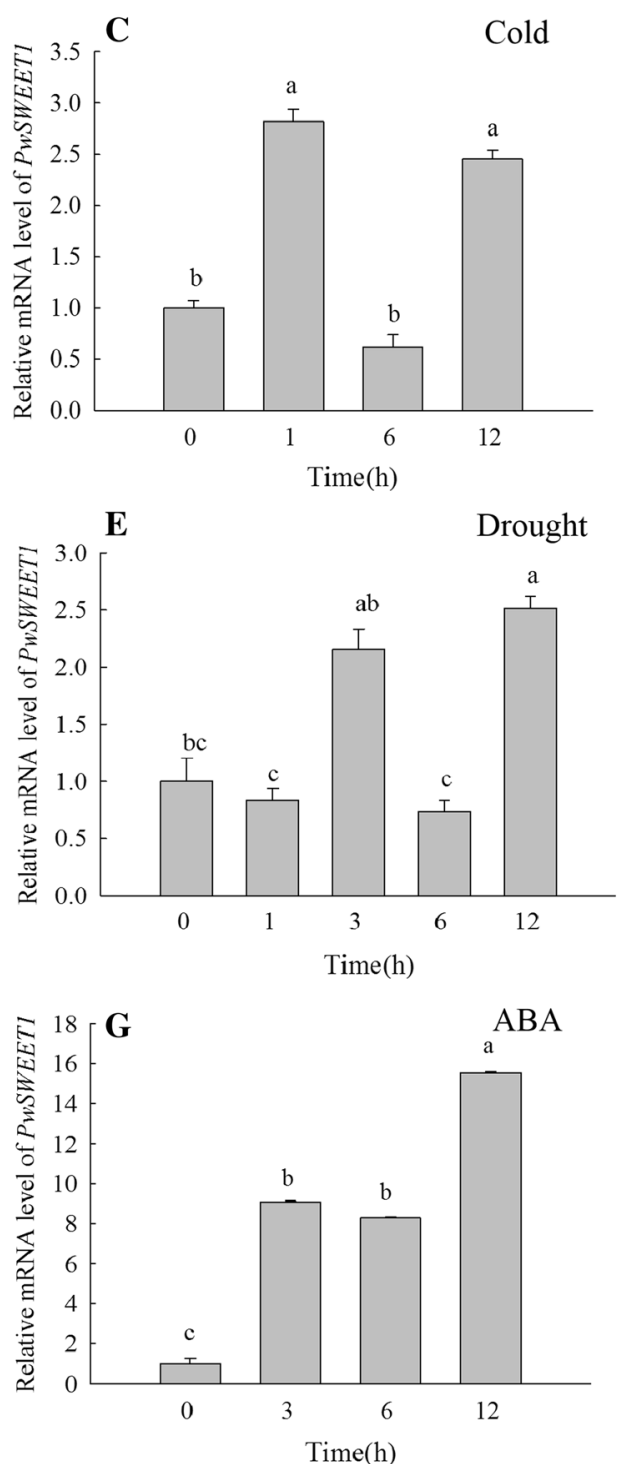

$\mathrm{NaCl}$

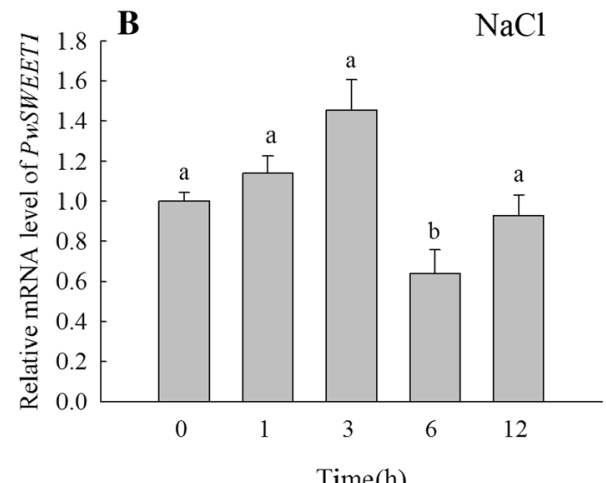

Heat
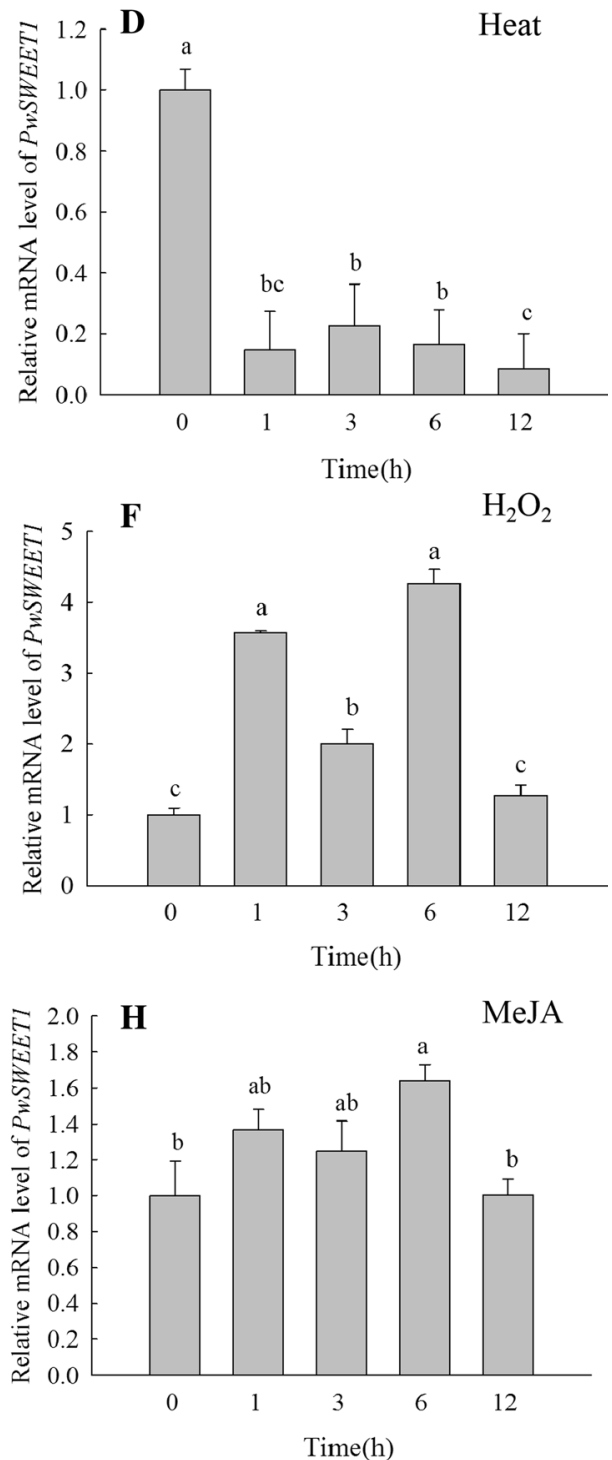

$0.1 \%(w / v) \mathrm{Ca}^{2+}$ and $0.1 \%(w / v) \mathrm{H}_{3} \mathrm{BO}_{3}$. As shown in Fig. $4 \mathrm{a}$ and $\mathrm{b}$, the percentage germination and pollen tube elongation were both repressed in the boron-amended medium after 12 and $24 \mathrm{~h}$. However, the tubes were significantly longer in the $\mathrm{Ca}^{2+}$-amended medium after $24 \mathrm{~h}$, and germination did not differ significantly from the control, despite the fact that $0.1 \%(\mathrm{w} / \mathrm{v}) \mathrm{Ca}^{2+}$ induced doubletipped tubes to fourfold compared to the control (Fig. S1A). PwSWEET1 expression was induced by boron, but not by $\mathrm{Ca}^{2+}$ after 12 and $24 \mathrm{~h}$ (Fig. 4c). 


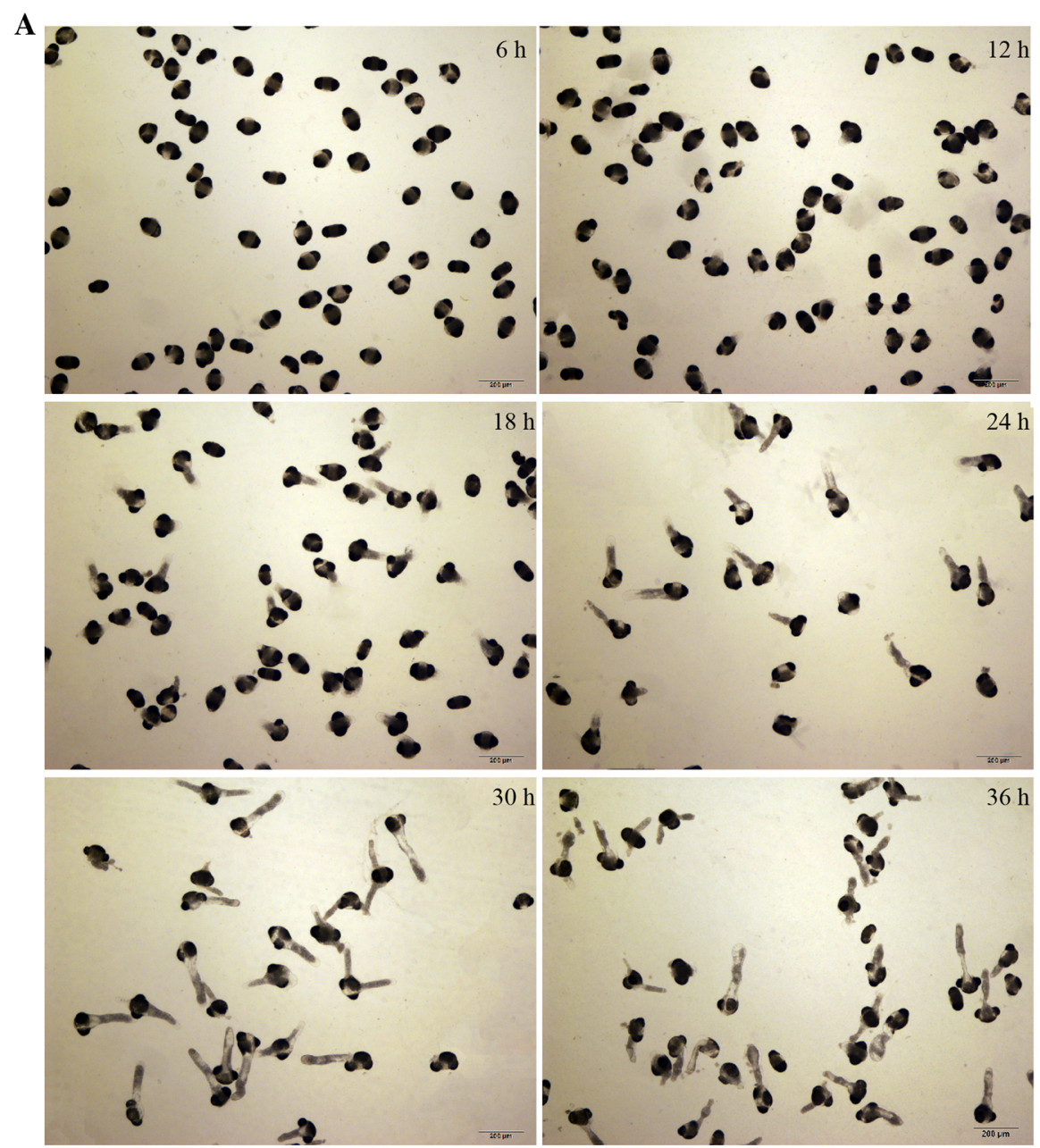

\section{$\mathbf{B}$}
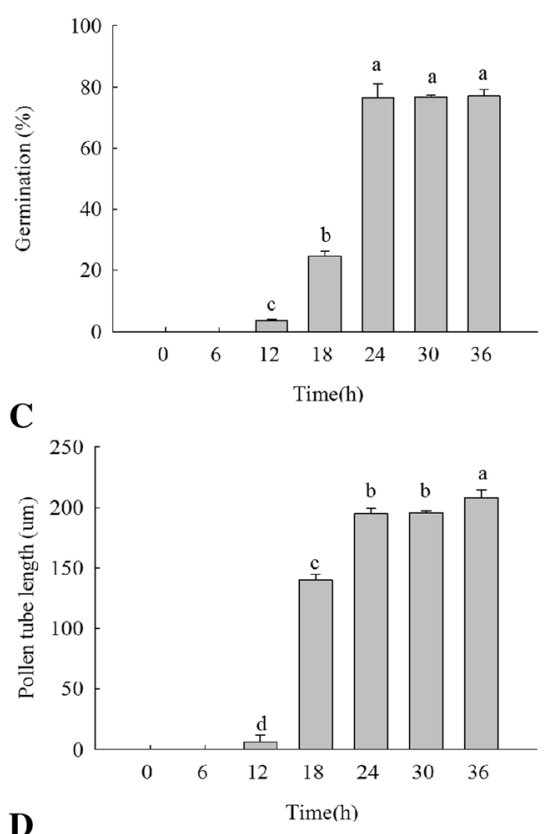

Fig. 3 Morphology and $P w S W E E T 1$ expression in germinating pollen of $P$. willsonii over time. a Light micrographs of pollen grains and pollen tubes, b percentage germination, c pollen tube lengths, $\mathbf{d}$ relative transcript levels of $P$ wSWEETI
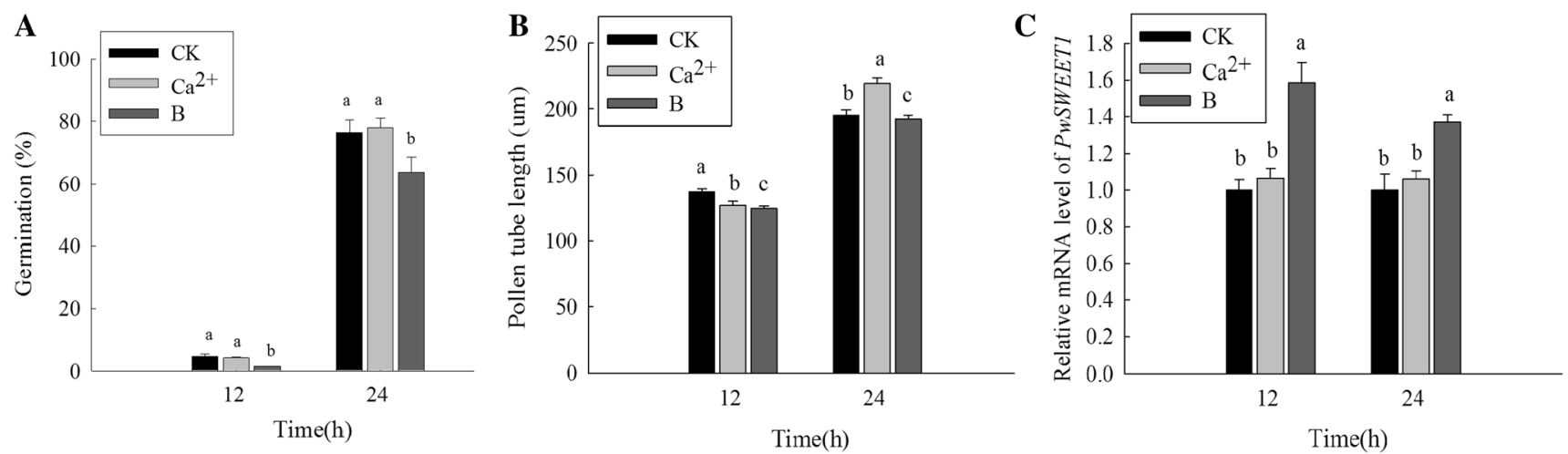

Fig. 4 Percentage germination, pollen tube elongation and PWSWEET1 expression after 12 and $24 \mathrm{~h}$ incubation in liquid medium amended with either of $0.1 \%(\mathrm{w} / \mathrm{v}) \mathrm{Ca}^{2+}$ and $0.1 \%(\mathrm{w} / \mathrm{v})$

$\mathrm{H}_{3} \mathrm{BO}_{3}$ (B). a Percentage germination. b Pollen tube lengths. c PWSWEET1 transcript levels in pollen. CK: control incubated in standard liquid medium

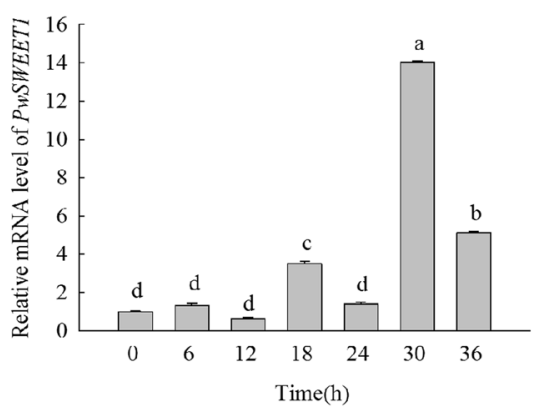


Sugar specificity and PwSWEET1 expression in pollen grains in different carbon sources

For pollen germination and tube growth in vitro, it tends to have a lower rate of double-tipped tubes when every sugar was at its best concentration (Fig. S1B). The best concentration for Suc, Glc, Gal, Mal and Fru was, respectively, $12 \%(\mathrm{w} / \mathrm{v}), 8 \%(\mathrm{w} / \mathrm{v}), 3 \%(\mathrm{w} / \mathrm{v}), 12 \%(\mathrm{w} / \mathrm{v})$ and $3 \%(\mathrm{w} / \mathrm{v})$, and Suc, Glc, and Mal yielded the best germination and longest pollen tubes (Fig. 5a, b). No pollen germination or tube growth occurred in medium containing mannose at any concentration we applied (not shown). Sucrose induced the highest expression of PwSWEET1, followed by Glc (Fig. 5c), suggesting that PwSWEET1 mainly transports sucrose and glucose.

\section{Heterologous expression of PwSWEET1 in yeast}

We tested whether PwSWEET1 is also a functional sugar transporter by transforming yeast strain EBY.VW4000 (Wieczorke et al. 1999), defective in hexose absorption, with the pDR196/PwSWEET1 fusion vector. All hexose transporter family genes (Hxt1-17) and five other transporter genes (Gal2, Stl1, Agt1, Mph2, Mph3) have been deleted in mutant strain EBY.VW4000, so the strain cannot grow on monosaccharides but can grow on maltose. Drop test indicated that EBY.VW4000 transformed either with pDR196/PwSWEET1 or the empty vector pDR196 can grow well on $2 \%(\mathrm{w} / \mathrm{v})$ Mal, whereas only pDR196/ PwSWEET1 transformants can grow on $2 \%$ (w/v) Glc (Fig. 6a).

To identify whether PwSWEET1 Glc transport is pHdependent, we tested Glc uptake by EBY.VW4000 transformed with pDR196/PwSWEET1 in medium supplemented with $2 \%(\mathrm{w} / \mathrm{v}) \mathrm{Mal}$ or $2 \%(\mathrm{w} / \mathrm{v}) \mathrm{Glc}$ at $\mathrm{pH} 4.0,5.0$, 6.0 and 7.0. The transformants grew well at each $\mathrm{pH}$ in medium containing either maltose or glucose, implying that PwSWEET1 is a pH-independent glucose transporter.

\section{Discussion and conclusions}

\section{SWEET1 in $P$. wilsonii is highly expressed in pollen}

SWEET gene family is widespread in eukaryotes and involved in many physiological processes and stress responses. In plants, it has a role in the nutrition of growing pollen tubes, senescence, phloem loading and plantpathogen interactions (Chen et al. 2010, 2012; Seo et al. 2011; Klemens et al. 2013; Sun et al. 2013). SWEET genes have been well studied in Arabidopsis and rice (Yang et al. 2006; Guan et al. 2008; Song et al. 2009; Chen et al. 2010, 2012; Chardon et al. 2013; Guo et al. 2014). However, its role in pollen germination and tube growth in plant, especially in coniferous species, has needed further study. Here, we described the functionally characterized $P$ WWEET1, a highly expressed gene in pollen in $P$. wilsonii. Phylogenetic analysis based on the amino acid sequences showed that PwSWEET1 was highly homologous with the four AtSWEET proteins and nine OsSWEET proteins. AtSWEET4, -5 and -7 function in glucose transport, and AtSWEET5 is pollen-specific (Engel et al. 2005; Chen et al. 2010). AtSWEET4 also mediates fructose transport during plant growth and development (Liu et al. 2016), and another member of clade 2 from Arabidopsis, AtSWEET8, which is also homologous to PwSWEET1, is a pollen-specific glucose transporter on the plasma membrane (Chen et al. 2010). In rice, OsSWEET5 encodes a galactose transporter (Zhou et al. 2014). The transmembrane helix (TM) prediction for PwSWEET1 and AtSWEET4, -5, -6, and -7 showed that PwSWEET1 and AtSWEET6 had 6 TMs, and the rest had 7 TMs (Fig. 1, Fig. S2).
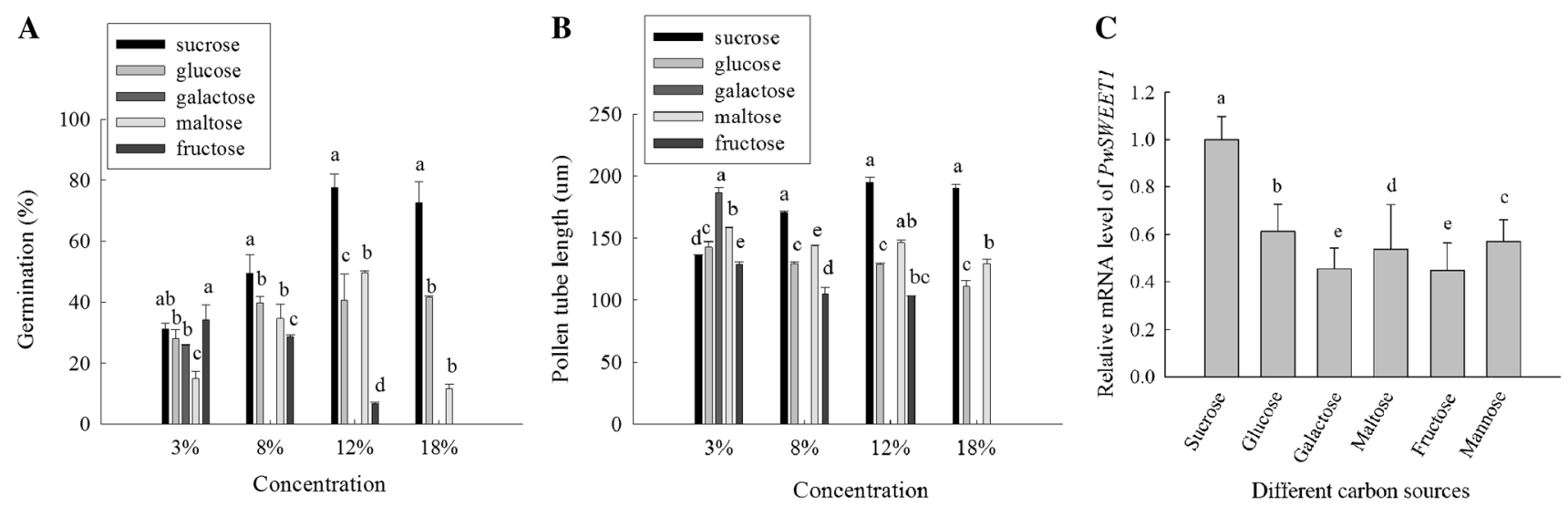

Fig. 5 Effect of different carbon sources on pollen germination, tube elongation and PwSWEET1 expression in vitro. a Germination. b Pollen tube lengths. c PwSWEET1 expression 
Fig. 6 Functional characterization of PwSWEET1 in yeast. a PwSWEET1 complemented glucose uptake in hexose transporter-deficient yeast EBY.VW4000 but pDR196 empty vector did not. b Complementation of glucose uptake deficiency of EBY.VW4000 by PwSWEET1 at different $\mathrm{pHs}$

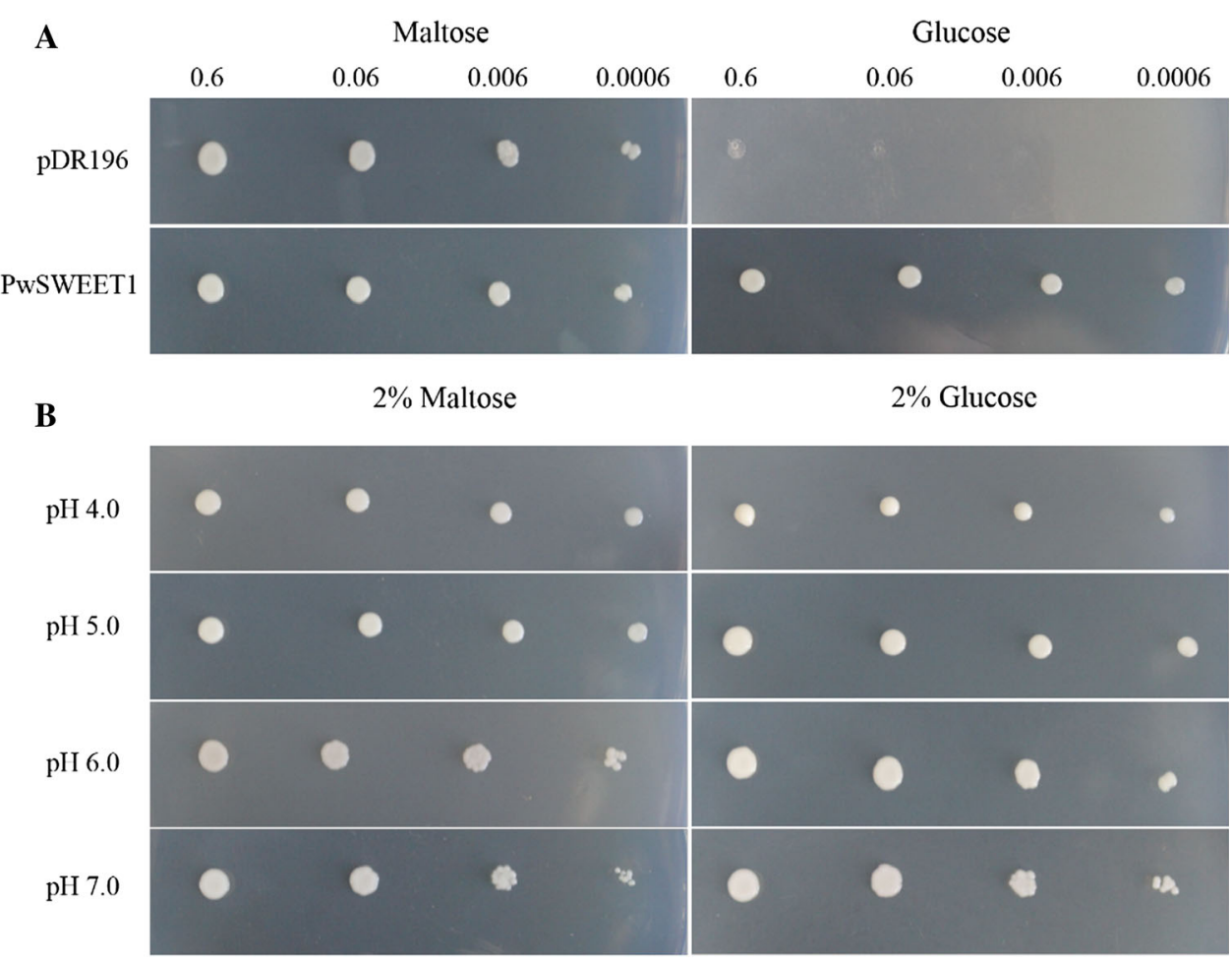

AtSWEET5 is expressed in mature, hydrated and germinating pollen (Engel et al. 2005). In the present tissuespecific assay, PwSWEET1 was shown to be pollen-specific, with levels in the pollen hundreds of times higher than in the cones, needles, stems and seeds (Fig. 2). PwSWEET1 expression was greatly induced at fast-growing stages of pollen tube development (Fig. 3) and enhanced further pollen grains exposed to $0.1 \% \mathrm{H}_{3} \mathrm{BO}_{3}$ for 12 and $24 \mathrm{~h}$ (Fig. 4). These results suggest that PwSWEET1 is very important in pollen germination and pollen tube growth and induced by boron.

\section{PwSWEET1 is a pH-independent glucose transporter}

In vitro pollen germination and pollen tube growth require sugar as an energy source, but different species require different sugars. Suc is the typical carbon source for pollen grains of most species (Okusaka and Hirastuka 2009; Cheng et al. 2015); however, many species use other sugars. Tobacco and A. thaliana pollen prefer Suc, whereas Petunia pollen can use Suc, Glc and Fru; cucumber pollen can use Suc, Glc and Gal, pear pollen uses Suc and Glc (Cheng et al. 2015; Lemoine et al. 1999; Okusaka and Hirastuka 2009; Scholz-Starke et al. 2003; Ylstra et al. 1998). Here, we found that $P$. wilsonii pollen tubes can grow on Suc, Glc and Mal at appropriate concentrations, but Suc was the most effective (Fig. 5).
PwSWEET1 expression also differs when pollen grains are incubated with different carbon sources; expression was highest with sucrose, followed by glucose (Fig. 5), suggesting SWEET1 differs in its ability to transport different types of sugars. The heterologous expression assay showed that PwSWEET1 functions in glucose transport and complemented glucose uptake deficiency of EBY.VW4000 (Fig. 6), similar to the homologous glucose transporters AtSWEET4, -5 and -7 (Chen et al. 2010). These data suggest that the structure is evolutionarily conserved, that SWEETs have diverse functions, and that the homologous genes in the SWEET family among different plant species have similar conserved functions and sugar preferences. Further, Glc uptake by yeast strain EBY.VW4000 transformed with pDR196/PwSWEET1 in medium supplemented with Mal or Glc at different $\mathrm{pHs}$ showed that transformants grew well at the four $\mathrm{pHs}$ either in Mal or Glc medium, proving that PwSWEET1 is a pH-independent glucose transporter. Although PwSWEET1 can transport glucose, we cannot preclude that it can also transport sucrose based on the function of homologs and the induction of PwSWEET1 by Suc and Gluc. It is not hard to imagine that there is an association among the preference of $P$. wilsonii pollen for Glc, the glucose transport ability of PwSWEET1 in yeast combination with the induction of PwSWEETI expression by glucose and sucrose, suggesting that $P W S W E E T 1$ is mainly probably responsible for Glu and Suc transport in pollen germination and pollen tube growth. 


\section{Possible function of the glucose transporter}

The RT-qPCR analysis showed that PwSWEET1 expression is enhanced under cold $\left(4^{\circ} \mathrm{C}\right), \mathrm{ABA}$, drought, and $\mathrm{H}_{2} \mathrm{O}_{2}$ treatment, but decreased by the heat treatment (Fig. 2). In contrast, $\mathrm{NaCl}$ and $\mathrm{MeJA}$ treatment caused no obvious changes, suggesting PwSWEETl is involved in multiple abiotic stresses. Previous studies showed that cold treatment at $4{ }^{\circ} \mathrm{C}$ can induce the accumulation of soluble sugars in plants and that sugar transporter genes can be regulated to balance the sugar content and ensure normal plant growth (Wormit et al. 2006; Klemens et al. 2013; Guo et al. 2014). For instance, after a cold treatment, overexpression of AtSWEET17 leads to decreased fructose accumulation (Chardon et al. 2013), and expression of AtSWEET16 is repressed (Klemens et al. 2013). Here we speculate that the accumulation of soluble sugars regulated by sugar transporters in plants also positively contributes to plant response to other such stresses as ABA, drought, and $\mathrm{H}_{2} \mathrm{O}_{2}$. Considering that PwSWEETI is specifically expressed in pollen and functions as a glucose transporter, suggesting PwSWEET1 plays a important role in sugar supply during the reproductive period and may take part in balancing sugar allocation in plants to ensure good growth since the accumulation of sugars can cause rapid changes in metabolism (Heineke et al. 1994). In fact, the AtSWEET5 gene, which is highly homologous to PwSWEET1 is localized specifically in the vegetative cell of pollen and may supply the generative cell with sugar (Engel et al. 2005).

Acknowledgements We thank Prof. Zhenxian Zhang of China Agricultural University for providing the hexose transporter-deficient yeast strain EBY.VW4000 and yeast expression vector pDR196. This work was supported by the grant from Agricultural Ministry of China (No. 2016ZX08009-003-002).

Open Access This article is distributed under the terms of the Creative Commons Attribution 4.0 International License (http://crea tivecommons.org/licenses/by/4.0/), which permits unrestricted use, distribution, and reproduction in any medium, provided you give appropriate credit to the original author(s) and the source, provide a link to the Creative Commons license, and indicate if changes were made.

\section{References}

Ap Rees T (1994) Plant physiology: virtue on both sides. Curr Biol 4(6):557-559

Buttner M, Sauer N (2000) Monosaccharide transporters in plants: structure, function and physiology. Biochim Biophys Acta 1465:263-274

Chardon F, Bedu M, Calenge F, Klemens PA, Spinner L, Clement G, Chietera G, Leran S, Ferrand M, Lacombe B, Londet O, Dinant S, Bellini C, Neuhaus E, Daniel-Vedele F, Krapp A (2013) Leaf fructose content is controlled by the vacuolar transporter SWEET17 in Arabidopsis. Curr Biol 23:697-702

Chen LQ (2014) SWEET sugar transporters for phloem transport and pathogen nutrition. New Phytol 201:1150-1155

Chen LQ, Hou BH, Lalonde S, Takanaga H, Hartung ML, Qu XQ, Guo WJ Kim JG, Underwood W, Chaudhuri B, Chermak D, Antony G, White FF, Somerville SC, Mudgett MB, Frommer WB (2010) Sugar transporters for intercellular exchange and nutrition of pathogens. Nature 468:527-532

Chen LQ, Qu XQ, Hou BH, Sosso D, Osorio S, Fernie AR, Frommer WB (2012) Sucrose efflux mediated by SWEET proteins as a key step for phloem transport. Science 335:207-211

Cheng J, Wang Z, Yao F, Gao L, Ma S, Sui X, Zhang Z (2015) Downregulating CsHT1, a cucumber pollen-specific hexose transporter, inhibits pollen germination, tube growth, and seed development. Plant Physiol 168:635-647

Engel ML, Holmes-Davis R, McCormick S (2005) Green sperm. Identification of male gamete promoters in Arabidopsis. Plant Physiol 138:2124-2133

Engelke T, Hirsche J, Roitsch T (2010) Anther-specific carbohydrate supply and restoration of metabolically engineered male sterility. J Exp Bot 61:2693-2706

Fan RC, Peng CC, Xu YH, Wang XF, Li Y, Shang Y, Du SY, Zhao R, Zhang XY, Zhang LY, Zhang DP (2009) Apple sucrose transporter SUT1 and sorbitol transporter SOT6 interact with cytochrome b5 to regulate their affinity for substrate sugars. Plant Physiol 150:1880-1901

Feng CY, Han JX, Han XX, Jiang J (2015) Genome-wide identification, phylogeny, and expression analysis of the SWEET gene family in tomato. Gene 573:261-272

Ferrari S, Galletti R, Denoux C, De Lorenzo G, Ausubel FM, Dewdney J (2007) Resistance to Botrytis cinerea induced in Arabidopsis by elicitors is independent of salicylic acid, ethylene, or jasmonate signaling but requires PHYTOALEXIN DEFICIENT3. Plant Physiol 144:367-379

Guan YF, Huang XY, Zhu J, Gao JF, Zhang HX, Yang ZN (2008) RUPTURED POLLEN GRAIN1, a member of the MtN3/saliva gene family, is crucial for exine pattern formation and cell integrity of microspores in Arabidopsis. Plant Physiol 147:852-863

Guo WJ, Nagy R, Chen HY, Pfrunder S, Yu YC, Santelia D, Frommer WB, Martinoia E (2014) SWEET17, a facil itative transporter, mediates fructose transport across the tonoplast of Arabidopsis roots and leaves. Plant Physiol 164:777-789

Hackel A, Schauer N, Carrari F, Fernie AR, Grimm B, Kunh C (2006) Sucrose transporter LeSUT1 and LeSUT2 inhibition affects tomato fruit development in different ways. Plant J 45:180-192

Heineke D, Wildenberger K, Sonnewald U, Willmitzer L, Heldt HW (1994) Accumulation of hexoses in leaf vacuoles: studies with transgenic tobacco plants expressing yeast-derived invertase in the cytosol, vacuole or apoplasm. Planta 194:29-33

Ji X, Shiran B, Wan J, Lewis DC, Jenkins CL, Condon AG, Richards RA, Dolferus R (2010) Importance of pre-anthesis anther sink strength for maintenance of grain number during reproductive stage water stress in wheat. Plant, Cell Environ 33:926-942

Johnson DA, Hill JP, Thomas MA (2006) The monosaccharide transporter gene family in land plants is ancient and shows differential subfamily expression and expansion across lineages. BMC Evol Biol 6:64

Klemens PA, Patzke K, Deitmer J, Spinner L, Le Hir R, Bellini C, Bedu M, Chardon F, Krapp A, Neuhaus H (2013) Overexpression of the vacuolar sugar carrier AtSWEET16 modifies germination, growth, and stress tolerance in Arabidopsis. Plant Physiol 163:1338-1352

Koch KE (1996) Carbohydrate-modulated gene expression in plants. Annu Rev Plant Physiol Plant Mol Biol 47:509-540 
Lazzaro BP (2005) Elevated polymorphism and divergence in the class C scavenger receptors of Drosophila melanogaster and D. simulans. Genetics 169(4):2023-2034

Lemoine R, Burkle L, Barker L, Sakr S, Kuhn C, Regnacq M, Gaillard C, Delrot S, Frommer WB (1999) Identification of a pollen-specific sucrose transporter-like protein NtSUT3 from tobacco. FEBS Lett 454:325-330

Liu X, Zhang Y, Yang C, Tian Z, Li J (2016) AtSWEET4, a hexose facilitator, mediates sugar transport to axial sinks and affects plant development. Sci Rep 6:24563

Ma H (2005) Molecular genetic analyses of microsporogenesis and microgametogenesis in flowering plants. Annu Rev Plant Biol 56:393-434

Marger MD, Saier MH Jr (1993) A major superfamily of transmembrane facilitators that catalyse uniport, symport and antiport. Trends Biochem Sci 18:13-20

McCormick S (2004) Control of male gametophyte development. Plant Cell 16(Suppl):142-153

Ogawa A, Kawashima C, Yamauchi A (2005) Sugar accumulation along the seminal root axis, as affected by osmotic stress in maize: a possible physiological basis for plastic lateral root development. Plant Prod Sci 8:173-180

Okusaka K, Hirastuka S (2009) Fructose inhibits pear pollen germination on agar medium without loss of viability. Sci Hortic 122:51-54

Patil G, Valliyodan B, Deshmukh R, Prince S, Nicander B, Zhao M, Sonah H, Song L, Lin L, Chaudhary J, Liu Y, Joshi T, Xu D, Nguyen HT (2015) Soybean (Glycine max) SWEET gene family: insights through comparative genomics, transcriptome profiling and whole genome re-sequence analysis. BMC Genom 16:520

Ramirez ML, Chulze SN, Magan N (2004) Impact of osmotic and matric water stress on germination, growth, mycelial water potentials and endogenous accumulation of sugars and sugar alcohols in Fusarium graminearum. Mycologia 96:470-478

Scholz-Starke J, Buttner M, Sauer N (2003) AtSTP6, a new pollenspecific $\mathrm{H}+$-monosaccharide symporter from Arabidopsis. Plant Physiol 131:70-77

Seo PJ, Park JM, Kang SK, Kim SG, Park CM (2011) An Arabidopsis senescence-associated protein SAG29 regulates cell viability under high salinity. Planta 233:189-200

Sivitz AB, Reinders A, Ward JM (2008) Arabidopsis sucrose transporter AtSUC1 is important for pollen germination and sucrose-induced anthocyanin accumulation. Plant Physiol 147:92-100

Slewinski TL (2011) Diverse functional roles of monosaccharide transporters and their homologs in vascular plants: a physiological perspective. Mol Plant 4:641-662
Song LF, Zou JJ, Zhang WZ, Wu WH, Wang Y (2009) Ion transporters involved in pollen germination and pollen tube tipgrowth. Plant Signal Behav 4:1193-1195

Sun MX, Huang XY, Yang J, Guan YF, Yang ZN (2013) Arabidopsis RPG1 is important for primexine deposition and functions redundantly with RPG2 for plant fertility at the late reproductive stage. Plant Reprod 26:83-91

Wieczorke R, Krampe S, Weierstall T, Freidel K, Hollenberg CP, Boels E (1999) Concurrent knock-out of at least 20 transporter genes is required to block uptake of hexoses in Saccharomyces cerevisiae. FEBS Lett 464:123-128

Wormit A, Trentmann O, Feifer I, Lohr C, Tjaden J, Meyer S, Schmidt U, Martinoia E, Neuhaus HE (2006) Molecular identification and physiological characterization of a novel monosaccharide transporter from Arabidopsis involved in vacuolar sugar transport. Plant Cell 18:3476-3490

Xu L, Wang WW, Su SC, Ma JX, Sun H, Fang ZF, Gao SQ, Tang YM, Zhao CP, Jiang T (2016) Cloning and expression analysis of a sugar transporter protein gene TaSWEET6 in Wheat. J Triticeae Crops 36(11):1009-1041

Yang B, Sugio A, White FF (2006) Os8N3 is a host diseasesusceptibility gene for bacterial blight of rice. Proc Natl Acad Sci USA 103:10503-10508

Ylstra B, Garrido D, Busscher J, van Tunen AJ (1998) Hexose transport in growing petunia pollen tubes and characterization of a pollen-specific, putative monosaccharide transporter. Plant Physiol 118:297-304

Ypsilos LK, Magan N (2004) Impact of water-stress and washing treatments on production, synthesis and retention of endogenous sugar alcohols and germinability of Metarhizium anisopliae blastospores. Mycol Res 108:1337-1345

Yu YL, Li YZ, Li LL, Lin JX, Zheng CC, Zhang LY (2009) Overexpression of PwTUA1, a pollen-specific tubulin gene, increases pollen tube elongation by altering the distribution of $\alpha$ tubulin and promoting vesicles transport. J Exp Bot 60:2737-2749

Yu Y, Li Y, Huang G, Meng Z, Zhang D, Wei J, Yan K, Zheng CC, Zhang LY (2011) PwHAP5, a CCAAT-binding transcription factor, interacts with PwFKBP12 and plays a role in pollen tube growth orientation in Picea wilsonii. J Exp Bot 62:4805-4817

Zhang D, Liu YJ, Li CJ, Cao YB, Zhang LY (2012) Construction of normolized cDNA library and analysis of corresponding EST sequences in Picea wilsonii. Biotechnol Bull 6:71-76

Zhou Y, Liu L, Huang WF, Yuan M, Zhou F, Li XH, Lin YJ (2014) Overexpression of OsSWEET5 in rice causes growth retardation and precocious senescence. Plos One 9(4):e94210

Publisher's Note Springer Nature remains neutral with regard to jurisdictional claims in published maps and institutional affiliations. 